\title{
IMPLEMENTAÇÃO DE SISTEMA DE GESTÃO DA QUALIDADE EM LABORATÓRIO: ESTUDO DE CASO EM UMA FÁBRICA DE ALIMENTAÇÃO ANIMAL
}

\author{
Patricia Freitas Pelozo Hespanhol ${ }^{1}$, Rafael Medeiros Hespanhol ${ }^{2}$ \\ ${ }^{1}$ Universidade Estadual Paulista - UNESP, Mestrado em Agronegócio e Desenvolvimento. ${ }^{2}$ Universidade do Oeste \\ Paulista - UNOESTE, Docente.
}

\section{RESUMO}

Sistemas de gestão da qualidade estabelecem procedimentos que orientam a execução de tarefas, sendo um fator essencial para a melhoria contínua dos processos. Desta forma, o presente artigo possui o objetivo de analisar o processo de implantação de um sistema de gestão de qualidade no laboratório de uma fábrica produtora de ração animal. Para isso, foi realizada a coleta de dados e levantamento de informações por meio de pesquisa-ação em um laboratório pertencente a uma fábrica do segmento. Para a implementação do sistema de gestão da qualidade, partiu-se das etapas propostas pelo ciclo PDCA. Os procedimentos adotados pelo laboratório melhoraram o desempenho das funções atribuídas aos funcionários do local e também diminuíram a probabilidade de erros no desenvolvimento das atividades diárias.

Palavras-chave: SGQ, Ciclo PDCA, Gestão da qualidade.

\section{QUALITY MANAGEMENT SYSTEM IMPLEMENTATION AT A LABORATORY: CASE STUDY IN AN ANIMAL FOOD FACTORY}

\begin{abstract}
Quality management systems establish procedures that guide tasks execution, being an essential factor for the continuous improvement of the processes. In this way, this paper aims to analyze the process of implanting a quality management system in the laboratory of an animal feed factory. For this, the data collection and information collection was carried out through action research in a laboratory belonging to a factory that produces animal feed. For the implementation of the quality management system, it was based on the stages proposed by the PDCA cycle. The procedures adopted by the laboratory improved the performance of the duties assigned to the site staff and also reduced the chances of errors in the development of daily activities.
\end{abstract}

Keywords: QMS, PDCA Cycle, Quality management

\section{INTRODUÇÃO}

Antes da Revolução Industrial, o artesão era uma espécie de especialista geral, que tinha domínio completo de todo o ciclo de produção, desde a concepção do produto até o pós-venda. Nesta época, havia proximidade entre o cliente e o artesão, que explicitava seus desejos e necessidades, e o artesão buscava atendê-los.
Essa busca por atender as necessidades dos cientes dava-se, pois o artesão sabia que a comercialização de seus produtos dependia muito de sua reputação de qualidade, que era comunicada boca a boca pelos clientes, tanto os satisfeitos, quanto os insatisfeitos. As abordagens de qualidade do artesão envolviam elementos como o atendimento às necessidades do cliente, 
por isso, o foco da gestão da qualidade se limitava ao produto, não tratando do processo (CARVALHO, 2013).

Chiroli (2016) explica que o artesanato representava uma produção de caráter familiar, em que todas as etapas eram realizadas em oficinas, desde o preparado da matéria prima até o produto final. Todas as etapas estavam, portanto, sob o cuidado de uma única pessoa, não havendo divisão do trabalho $\mathrm{e}$ especialização.

Com a Revolução Industrial, os sistemas produtivos se modificaram e a customização passou a ser substituída pela padronização e produção em larga escala. Quando o sistema de produção em larga escala passou a tomar conta das fábricas, o conceito de controle de qualidade passou por diversas modificações e aprimoramento.

No século $X X$, houve grande evolução do conceito de controle de qualidade, que teve a influência do sistema de linha de montagem desenvolvido por Ford, que adotou um sistema padronizado de medida para todas as peças produzidas. Em 1924, houve um novo salto no conceito de controle da qualidade, quando Walter A. Shewhart criou gráfico de controle, que acabou por implantar conceitos de estatística à realidade produtiva. Logo depois, Shewhart propôs o ciclo PDCA, que direcionaria as atividades de análise e solução de problemas (CARVALHO, 2013).

O ciclo PDCA, proposto por Shewhart, constitui a base dos modelos de sistema de gestão da qualidade, sendo considerada uma metodologia com a função de auxiliar na análise de problemas organizacionais. O método mostrase eficiente, visto que existem poucos instrumentos que conduzem a ações sistemáticas e a obtenção de melhores resultados (QUINQUIOLO, 2002; CHIROLI, 2016).

$\mathrm{O}$ método PDCA originou-se nos Estados Unidos como um ciclo de controle estatístico do processo e foi popularizado pelo especialista em qualidade W. Edwards Deming, que ficou mundialmente conhecido por aplicar o método nos conceitos de qualidade no Japão (SOUZA, 1997).

O ciclo PDCA é um método gerencial de tomada de decisão que é utilizado para garantir o alcance de metas organizacionais. Quando o processo produtivo possui um resoltado indesejado, a organização identifica o problema e o clico PDCA é utilizado como um método de tomada de decisões para a resolução de problemas organizacionais (WERKEMA, 1995; FONSECA; MIYAKE, 2006).

O PDCA busca a melhoria contínua e garantia do funcionamento dos processos por meio de quatro etapas, sendo elas o planejamento, a execução, a checagem e ação (CHIROLI, 2016).

A etapa de planejamento envolve o processo no qual devem ser estabelecidas as diretrizes e metas a serem atingidas, assim como os caminhos que devem ser percorridos em buscas desse objetivo. Na etapa de execução, acontece o treinamento dos envolvidos, a execução das atividades planejadas na etapa anterior e coleta de dados importantes para as etapas seguintes. A checagem é a terceira etapa do desenvolvimento do ciclo PDCA, sendo nesta etapa que os trabalhos executados são monitorados para identificar se os objetivos estão sendo atingidos e descrever os resultados alcançados. A última etapa é a ação, que envolve a análise dos resultados obtidos na etapa anterior e, se houver sucesso na execução de acordo com o planejado, padroniza-se o processo, do contrário, é preciso retornar a fase de planejamento buscando um novo método para solucionar o problema (CHIROLI, 2016).

A utilização do ciclo PDCA é uma forma de inserir qualidade final no produto por meio da execução dessas etapas. No entanto, a definição de termo qualidade é muito discutido e considerado subjetivo por diversos autores (GOMES, 2004; CARVALHO, 2013; CHIROLI, 2016). Um produto é considerado como de qualidade quando cumpre a sua função da forma como o cliente deseja. Considera-se que um serviço possui qualidade atende ou até mesmo supera as expectativas (GOMES, 2004).

Chiroli (2016) afirma que existe uma série de definições para o termo qualidade, principalmente no sentido de determinar adequação ao uso, porém o conceito desse termo evoluiu. Para Chiroli (2016), a qualidade significa muito mais do que a integridade de um produto manufaturado, tratando-se de um conceito complexo e abrangente, representando uma filosofia, um sistema de metodologias e práticas e compromisso com a excelência de negócios que abrange todas as áreas, indivíduos e questões das organizações.

Carvalho (2013) trata a qualidade em cinco abordagens distintas. A primeira abordagem trata da qualidade como 
transcendental, que se trata de características de excelência inata e universalmente reconhecível. A segunda abordagem trata da qualidade baseada no produto que, neste caso, é considerada como uma variável precisa e mensurável, procedente dos atributos do produto. A qualidade baseada no usuário é a terceira abordagem proposta, sendo uma variável subjetiva, em que produtos de melhor qualidade são aqueles atendem melhor aos desejos dos consumidores. A quarta abordagem de qualidade é baseada na produção, sendo uma variável precisa e mensurável, oriunda do grau de conformidade do planejado com o executado, no caso, uma abordagem com ênfase em ferramentas estatísticas (controle do processo). A última abordagem é baseada no valor, sendo de difícil aplicação, pois mistura os conceitos de excelência e valor, destacando o trade-off qualidade versus preço.

A qualidade do produto é a capacidade deste em atender as necessidade e expectativas do cliente, sendo que para o produto tenha esta característica, deve ser produzido de maneira adequada, dentro de padrões determinados, atendendo às expectativas do cliente. Deste modo, o produto precisa ser produzido dentro de um sistema chamado de Sistema de Gestão da Qualidade (OLIVARES, 2008).

O Sistema de Gestão da Qualidade atua em todos os níveis e funções de uma organização, desde os clientes internos até os externos. Para implementar um sistema de gestão de qualidade em laboratórios, é necessário criar procedimentos e padronizar adequadamente todas as atividades laboratoriais (OLIVERES, 2008).

O processo de padronização das atividades dos laboratórios de ensaio e calibração iniciou-se no Brasil em 2001, a partir da publicação da ABNT NBR USO/IEC 17025. Atualmente, se encontra em vigor a NBR ISSO/IEC 17025:2005 que passou a vigorar a partir de 2005. No entanto, internacionalmente esse processo teve início em 1978, a partir da norma ISO/IEC 14025 que estabelecia requisitos gerais para a competência de laboratórios de ensaio e calibração, oficialmente publicada em 2000.

Considerando os procedimentos envolvendo um laboratório que realiza análise de matérias primas e produtos acabado em uma fábrica de alimentação animal, o presente trabalho pretende responder a seguinte questão: é possível implementar um sistema de gestão de qualidade no laboratório pertencente a uma fábrica produtora de ração animal?

Os Sistemas de Gestão da Qualidade (SGQ) determinam uma estrutura organizacional que garante a qualidade por meio de os recursos, procedimentos operacionais e responsabilidades estabelecidas. Os procedimentos do SGQ devem orientar na execução de determinadas tarefas, sendo um fator essencial para a melhoria contínua dos processos, manutenção e controle dos registros das atividades executadas (PIMENTA, 2015). Neste contexto, o trabalho tem como objetivo de analisar o processo de implantação um sistema de gestão de qualidade no laboratório de uma fábrica produtora de ração animal.

\section{METODOLOGIA}

O presente trabalho possui abordagem qualitativa, que segundo Ludke e André (1986), possui como características os dados serem predominantemente descritivos. Nessa abordagem, é muito rica a descrição de pessoas, situações e acontecimentos no material obtido por meio de entrevistas, depoimentos ou observação.

Para o desenvolvimento da pesquisa e coleta de dados e informações, realizada no ano de 2017 , foi adotada a metodologia de pesquisaação. Martins (2010) explica que na pesquisaação o pesquisador tem um grande envolvimento com os indivíduos e a organização pesquisada, fazendo parte da equipe que realiza uma mudança organizacional por meio da pesquisa. Desta forma, um dos autores deste trabalho fazia parte da equipe que trabalhava na implementação do sistema de gestão da qualidade na fábrica, tendo assim, total acesso aos dados e controles desenvolvidos durante o processo. Conforme as planilhas e dados eram coletados no dia a dia da organização, tudo era registrado e analisado.

A implementação do sistema de gestão da qualidade foi realizada a partir da metodologia proposta pelo Ciclo PDCA, seguindo suas etapas de planejamento, execução, verificação e controle, que serão detalhadas nos resultados deste trabalho.

\section{RESULTADOS}

Com o objetivo de controlar e garantir a qualidade na fabricação de produtos de alimentação animal, a organização pesquisada estruturou um laboratório de controle de 
qualidade, exerce papel fundamental no sistema de gestão. É por meio das análises realizadas no laboratório que se torna possível o efetivo controle das matérias primas, o controle da água, a validação de processos, a validação da eficácia das limpezas, o controle de produtos acabados, além das atividades de pesquisa, respostas às reclamações dos clientes e decisões de recall. Atividades de laboratórios têm seu sistema de gestão definidos e os requisitos especificados na norma NBR/ISO/IEC 17025, que estão de acordo com os requisitos da ISO 9000:2000.

Seguindo Olivares (2008), para implementar um sistema de gestão de qualidade em laboratórios é necessário criar procedimentos e padronizar adequadamente todas as atividades laboratoriais. No processo de iniciação da implementação do sistema de gestão da qualidade, é preciso estabelecer as documentações necessárias que devem conter políticas, manuais técnicos e administrativos, instruções de trabalho e de operação de equipamentos, planos de calibração e manutenção preventiva de equipamentos, registros dos dados brutos, programas de treinamentos de pessoal e documentos externos como legislações, normas técnicas, especificações de clientes, certificados de calibração, certificados de materiais de referencia e softwares (OLIVEIRA, 2004).

Para implementar o sistema de controle de qualidade na organização estudada, partiu-se do método do Ciclo PDCA. A primeira etapa, seguindo a literatura disponível Werkema (1995), Fonseca e Miyake (2006) e Chiroli (2016), foi realizado o planejamento de como essa implementação aconteceria, em que se focou em estabelecer as documentações necessárias. Nesta etapa, planejou-se a criação dos seguintes itens: planilhas de registro de dados dos resultados de análises de matéria prima; planilhas de registro de dados dos resultados de análises de produto acabado; procedimentos Operacionais Padrões (POPs) de todas as análises realizadas no laboratório; relatórios de Não Conformidade.

Na segunda etapa do ciclo PDCA, que é a fase da execução, iniciou-se o desenvolvimento das planilhas e a inserção dos dados referentes aos resultados obtidos nas análises laboratoriais para registra-las. No banco de dados das matérias primas, implementou-se o registro de resultados das análises com informações como: produto, fornecedor, lote interno, data da chegada, data de validade, resultados de proteína, extrato etéreo, matéria mineral, fósforo e umidade. Acrescentou-se uma coluna referente a soma centesimal, uma forma de garantir resultados corretos, tendo como base a teoria de que a soma dos resultados deve ser próxima do $100 \%$. No status do lote, identificou-se a informação de que o lote já foi consumido, está em aberto ou está fechado aguardando ser utilizado.

Cada matéria prima passou a ter sua aba específica de resultados, como demonstra no Quadro 1. 
Quadro 1. Registro de dados dos resultados de análises de matéria prima

\begin{tabular}{|c|c|c|c|c|c|c|c|c|c|c|c|c|}
\hline Produto & $\begin{array}{l}\text { Forne- } \\
\text { cedor }\end{array}$ & Lote & Chegada & Validade & $\begin{array}{l}\text { Proteína } \\
\text { mín. 55,0 }\end{array}$ & $\begin{array}{c}\text { Extrato } \\
\text { etéreo } \\
\text { mín. } \mathbf{8 , 0}\end{array}$ & $\begin{array}{c}\text { Mat. } \\
\text { Mineral } \\
\text { máx. 25,0 }\end{array}$ & $\begin{array}{l}\text { Cálcio } \\
\text { máx. 12,0 }\end{array}$ & $\begin{array}{l}\text { Fósforo } \\
\text { mín. 4,0 }\end{array}$ & $\begin{array}{l}\text { Umidade } \\
\text { máx. 8,0 - }\end{array}$ & $\begin{array}{c}\text { Soma } \\
\text { centesimal }\end{array}$ & Status do lote \\
\hline Padrão & & & & & 55 & 8 & 25 & 12 & 4 & 8 & & \\
\hline $\begin{array}{l}\text { Farinha de } \\
\text { peixe }\end{array}$ & $X$ & $\begin{array}{l}\mathrm{FP} \\
01 / 16\end{array}$ & 02/01/16 & 02/06/16 & 63,68 & 8,71 & 26,24 & 9,77 & 4,03 & 5,1 & 103,73 & ACABOU \\
\hline $\begin{array}{l}\text { Farinha de } \\
\text { peixe }\end{array}$ & Z & $\begin{array}{l}\text { FP } \\
03 / 16\end{array}$ & 04/01/16 & 04/06/16 & 62,54 & 7,41 & 25,4 & 14,13 & 4,2 & 2,8 & 98,15 & ACABOU \\
\hline $\begin{array}{l}\text { Farinha de } \\
\text { peixe }\end{array}$ & Z & $\begin{array}{l}\text { FP } \\
04 / 16\end{array}$ & 06/01/16 & 05/06/16 & 61,49 & 8,10 & 24,61 & 7,91 & 3,44 & 3,8 & 89,9 & ACABOU \\
\hline $\begin{array}{l}\text { Farinha de } \\
\text { peixe }\end{array}$ & $Y$ & $\begin{array}{l}\text { FP } \\
05 / 16\end{array}$ & $10 / 01 / 16$ & $10 / 06 / 16$ & 58,15 & 7,33 & 25,49 & 10,19 & 3,16 & 4,2 & 95,17 & ACABOU \\
\hline $\begin{array}{l}\text { Farinha de } \\
\text { peixe }\end{array}$ & Z & $\begin{array}{l}\text { FP06/1 } \\
6\end{array}$ & $12 / 01 / 16$ & $12 / 06 / 16$ & 59,63 & 12,02 & 23,6 & 8,02 & 3,69 & 4 & 99,25 & USO \\
\hline $\begin{array}{l}\text { Farinha de } \\
\text { peixe }\end{array}$ & $x$ & $\begin{array}{l}\text { FP } \\
07 / 16\end{array}$ & $15 / 01 / 16$ & $15 / 06 / 16$ & 57,5 & 12,13 & 23,85 & 9,03 & 4,3 & 5,3 & 98,78 & FECHADO \\
\hline
\end{tabular}

Fonte: Os autores (2019). 
Assim como na planilha de matéria prima, cada produto acabado passou a possuir uma aba na planilha de registro, demonstrada no Quadro 2.

Quadro 2. Registro de dados dos resultados de análises de produto acabado.

\begin{tabular}{|c|c|c|c|c|c|c|c|c|c|}
\hline Produto & $\begin{array}{l}\text { Data de } \\
\text { fabricação }\end{array}$ & Lote & $\begin{array}{l}\text { Proteín } \\
\text { a }\end{array}$ & \begin{tabular}{|l} 
Extrat \\
o \\
etéreo
\end{tabular} & $\begin{array}{l}\text { Mat. } \\
\text { Mineral }\end{array}$ & $\begin{array}{l}\text { Fibra } \\
\text { bruta }\end{array}$ & Cálcio & Fósforo & Umidade \\
\hline \multicolumn{3}{|c|}{$\begin{array}{l}\text { Valores nutricionais esperados } \\
\text { na formula }\end{array}$} & $32 \%$ & $6 \%$ & $\begin{array}{l}7,5 \mathrm{a} \\
9,0 \%\end{array}$ & $\begin{array}{l}3,5 \mathrm{a} \\
4,0 \%\end{array}$ & $\begin{array}{l}1,8 \mathrm{a} \\
2,0 \%\end{array}$ & 1,0 a $1,2 \%$ & 8,0 a $9,0 \%$ \\
\hline \multicolumn{3}{|c|}{ Níveis de garantia } & $\begin{array}{l}\text { Mín. } \\
32 \%\end{array}$ & $\begin{array}{l}\text { Mín. } \\
6 \%\end{array}$ & $\begin{array}{l}\text { Max. } \\
13 \%\end{array}$ & \begin{tabular}{|l} 
Max. \\
$7 \%$ \\
\end{tabular} & $\begin{array}{l}\text { Max. } \\
3,0 \% \\
\end{array}$ & $\begin{array}{l}\text { Mín. } \\
1,0 \% \\
\end{array}$ & $\begin{array}{l}\text { Max. } \\
13,0\end{array}$ \\
\hline$x$ & \begin{tabular}{|l|}
$03 / 08 / 201$ \\
5 \\
\end{tabular} & $302 / 15$ & 32,17 & 7,51 & 8,23 & 3,79 & 2,21 & 1,1 & 9,5 \\
\hline$x$ & \begin{tabular}{|l|}
$10 / 08 / 201$ \\
5 \\
\end{tabular} & $313 / 15$ & 33,38 & 7,06 & 7,97 & 2,71 & 1,9 & 1,3 & 8,2 \\
\hline$x$ & \begin{tabular}{|l|}
$17 / 08 / 201$ \\
5 \\
\end{tabular} & $323 / 15$ & 32,21 & 6,11 & 7,17 & 3,9 & 1,87 & 1,2 & 9,1 \\
\hline$x$ & $\begin{array}{l}24 / 08 / 201 \\
5\end{array}$ & $343 / 15$ & 32,33 & 7,37 & 8,03 & 3,2 & 2,01 & 1,1 & 8,5 \\
\hline
\end{tabular}

Fonte: Os autores (2019).

Dando continuidade ao processo de implementação do sistema de gestão de qualidade no laboratório, foram criados os chamados "Procedimentos Operacionais Padrões" (POP), que se referem à descrição de como cada análise realizada dentro do laboratório deve ser feita, sendo classificados da seguinte forma:

- POP CQ 01 - Acidez;

- POP CQ 02 - Índice de Fibra Bruta;

- POP CQ 03 - Índice de Matéria Mineral;

- POP CQ 04 - Índice de Cálcio por Oxidimetria;

- POP CQ 05 - Índice de Umidade por estufa;

- POP CQ 06 - Índice de Proteína Bruta;

- POP CQ 07 - Determinação de Extrato Etéreo Hidrólise Ácida;

- POP CQ 08 - Determinação de Gordura ou Extrato Etéreo;

- POP CQ 09 - Índice de Peróxido;

- POP CQ 10 - Teste de Éber;

- POP CQ 11 - Índice de atividade de água;

- POP CQ 12 - Umidade de lenha;

- POP CQ 13 - Teste de Aflatoxina;

- POP CQ 14 - Densidade;

- POP CQ 15 - Análise de Fósforo;
- POP CQ 16 - Descarte de Resíduo do laboratório;

- POP CQ 17 - Lavagem e esterilização das vidrarias;

- POP CQ 18 - Tratamento de água para análise;

- POP CQ 19 - Amostragem de produtos para descarga;

- POP CQ 20 - Registro e identificação de soluções preparadas.

Em cada um dos POPs criados, foram descritas de forma detalha as etapas para a realização da atividade especificada. 0 Procedimento Operacional Padrão é desenvolvido com o objetivo de descrever a sequencia para garantir o resultado esperado de uma tarefa e tornar possível que a atividade seja realizada por qualquer pessoa (GUERRERO, BECCARIA, TREVIZAN, 2008).

Logo após o desenvolvimento dos POPs, foi criado o procedimento chamado de "Controle de Não Conformidades", em que ao ser identificado qualquer produto com especificações diferentes das padronizadas pela empresa durante as rotinas de análises, o responsável pela identificação deve comunicar a Gerência da Qualidade verbalmente e por registo escrito sobre o fato. Ao desenvolver os produtos, a organização definiu, com base em consultoria 
feita com especialista em nutrição animal, quais deveriam ser os padrões ideais para cada de seus produtos, o que inclui características como tamanho, cor, densidade, granometria, níveis nutricionais, entre outros aspectos que influenciam na qualidade do produto. Desta forma, o "Controle de Não Conformidades" foi criado para relatar qualquer desvio de padrão identificado nos produtos, e também nas matérias primas recebidas diariamente na fábrica, pois para garantir a qualidade e níveis nutricionais do produto final, cada matéria prima também teve seus padrões definidos.
Quando o controle de qualidade relata uma não conformidade, fica a critério da gerência a necessidade da aplicação de ação corretiva ou a interrupção de serviços. Como exemplo, pode-se relatar um caso em que, ao ser identificada uma carga de matéria prima com índices fora dos padrões exigidos pela empresa, o controle de qualidade não libera o descarregamento da mesma, aconselhando à gerencia a devolução da carga ao fornecedor, sendo a decisão final de exclusividade da gerencia.

Quadro 3. Modelo do Relatório de não conformidade

\begin{tabular}{|l|l|}
\hline \multicolumn{2}{|c|}{ RELATÓRIO DE NÃO CONFORMIDADE } \\
\hline $\mathrm{N}:$ & Produto: \\
\hline Data: & Quantidade: \\
\hline Lote: & Data de fabricação: \\
\hline
\end{tabular}

\begin{tabular}{|l|}
\hline Descrição da ocorrência: \\
\hline Parecer do Controle de Qualidade: \\
\hline \\
\hline Ações corretivas: \\
\hline \\
\hline RESPONSÁVEL: \\
\hline
\end{tabular}

Fonte: Os autores (2019).

Após a implementação do sistema de gestão de qualidade no laboratório, as atividades passaram a ser checadas pela analista responsável pelo mesmo, como determinado pela terceira etapa do ciclo PDCA. Esse analista passou a conferir diariamente o desenvolvimento das atividades e principalmente se os registros das mesmas estão sendo feitos de forma correta.

Com essa etapa de checagem foi possível identificar algumas falhas existentes no sistema, como informações que poderiam ser adicionadas às planilhas de registros e no relatório de não conformidades. Desta forma, para a próxima fase do ciclo PDCA, o controle de qualidade propôs propor a empresa ajustar e melhorar o sistema. Neste caso, o ciclo PDCA deve se iniciar novamente, sendo feito $o$ processo de planejamento da mudança para depois colocá-las em práticas e controlá-las.

\section{DISCUSSÃO}

Antes da implementação do sistema de gestão da qualidade pela fábrica, alguns desses processos já ocorriam, porém de forma informal e sem registros. Sendo assim, ao longo do tempo, quando a organização recebia devoluções de produtos ou quando identificação qualquer tipo de problema em lotes fabricados anteriormente, já não era possível identificar as causas dos problemas, ou até mesmo se ele foi de responsabilidade da própria fábrica ou se o problema ocorreu fora da mesma.

Um dos principais benefícios adquiridos com a implementação do sistema de gestão da qualidade, foi a possibilidade de rastreamento em ocorrências de reclamações e devoluções de produtos.

Ao receber uma devolução ou reclamação, a primeira ação do responsável pelo controle de qualidade passou a ser a identificação do lote envolvido e busca por informações se ocorreu alguma não conformidade com aquele lote e também se os índices nutricionais registrados nas planilhas de matéria prima e produto final estavam de acordo com o padronizado pela empresa. Desta forma, o atendimento ao cliente e resolução de problemas passou a acontecer de forma mais ágil, pois a 
empresa passou a identificar sua parcela de responsabilidade no problema detectado pelos clientes. Uma devolução, que antes da implementação do controle de qualidade, demorava cerca de 5 dias para ser aprovada ou rejeitada, passou a ser desenvolvida em apenas dois, o que refletiu até na satisfação dos clientes com o serviço prestado.

A implementação do sistema de gestão da qualidade também refletiu de forma significativa nas tarefas diárias desenvolvidas pelos funcionários do controle da qualidade da organização. Com a elaboração dos Procedimentos Operacionais Padrões, houve uma redução da dependência dos analistas do laboratório em relação aos seus superiores. Os superiores passaram a ensinar as funções, acompanhar as análises e apenas seguindo os POPs, os analistas se tornavam capazes de realizar as atividades de forma independente mais rápido, sendo de responsabilidade dos superiores apenas fazer vistorias periódicas para atestar a qualidade das análises realizadas. Portanto, os POPs proporcionaram economia em treinamentos para a fábrica, considerando que a quantidade de treinamentos para os funcionários que começavam a trabalhar no laboratório passou a ser menor do que quando não existiam os POPs. Antes da elaboração, o analista do laboratório passava por um mês de treinamento para garantir autonomia nas análises, sendo que após a implementação esse período foi reduzido para duas semanas.

\section{CONSIDERAÇÕES FINAIS}

O trabalho atingiu o objetivo de analisar a implementação um sistema de gestão de qualidade em um laboratório pertencente a uma fábrica produtora de ração animal. Os procedimentos adotados pelo laboratório buscaram melhorar o desempenho das funções atribuídas aos funcionários do local e também diminuir as chances de erros no desenvolvimento das atividades diárias. Antes da implementação, ao chegar um funcionário novo no laboratório, o tempo de adaptação era bem maior, pois não havia os POPs que explicava detalhadamente como as atividades deviam ser desenvolvidas por ele.

Essa implementação acabou se traduzindo em inúmeras vantagens. Os registros criados para as matérias primas e produtos acabados auxiliaram significativamente no trabalho, visto que antes as anotações eram feitas em papéis, sem forma padronizada, em que cada analista adotava seu próprio modelo, o que dificultava o entendimento quando o mesmo não estava presente no laboratório. Com os registros dos indicies das matérias primas e produtos finais e a elaboração do controle de não conformidade, a resolução de problemas com as devoluções e reclamações dos clientes passou a acontecer em menos da metade do tempo que ocorria antes e com os POPs proporcionaram uma queda na necessidade de treinamento dos analistas do laboratório e diminuição das chances de erros em análises.

O controle de não conformidades proporcionou uma segurança maior ao controle de qualidade, pois se tornou possível rastrear todas as não conformidades que já aconteceram no laboratório, além de ser uma forma de formalizar o repasse de informações para a alta direção da fábrica, visto que todas as não conformidades encontradas eram encaminhadas para os mesmos.

\section{REFERÊNCIAS}

CARVALHO M. Histórico da Gestão da Qualidade. In: CARVALHO, M.; PALADINI, E. Gestão da qualidade: teoria e casos. Rio de Janeiro: Elsevier Brasil, 2013.

\section{CHIROLI D. M. G. Avaliação dos sistemas de} qualidade. CURITIBA: Intersaberes, 2016.

FONSECA, A. V. M.; MIYAKE, D. I. Uma análise sobre o Ciclo PDCA como um método para solução de problemas da qualidade. In: Encontro Nacional de Engenharia de Produção, 26., 2006, Fortaleza. Anais eletrônicos [...]. Fortaleza: ENEGEP, 2006. Disponível em: http://www.abepro.org.br/biblioteca/ENEGEP20 06_TR470319_8411.pdf. Acesso em: 25 jan. 2019.

GOMES, P. A evolução do conceito de qualidade: dos bens manufacturados aos serviços de informação. Cadernos Bad, n. 2, 2004.

GUERRERO, G. P.; BECCARIA, L. M.; TREVIZAN, M. A. Procedimento operacional padrão: utilização na assistência de enfermagem em serviços hospitalares. Revista Latino-Americana de Enfermagem, v. 16, n. 6, p. 966-972, 2008. https://doi.org/10.1590/S0104$\underline{11692008000600005}$ 
LUDKE, M.; ANDRÉ, M. E. D. A. Pesquisa em Educação: abordagens qualitativas. São Paulo: E.P.U., 1986.

MARTINS, R. A. Abordagens quantitativa e qualitativa. In: MIGUEL, P. A. M (org.). Metodologia de pesquisa em engenharia de produção e gestão de operações. Rio de Janeiro: Elsevier, 2010, p.45-61.

OLIVARES, I. R. B. Gestão da Qualidade em Laboratórios. 2008. São Paulo. 172 p. Notas de aula. 2008.

OLIVEIRA, O. J. Gestão da qualidade: introdução à história e fundamentos. In: Gestão da qualidade: tópicos avançados. São Paulo: Pioneira Thomson Learning, p. 3-20, 2004.

PIMENTA, E. B. Gestão da qualidade. 2015. $13 \mathrm{f}$. Trabalho de conclusão de curso (Administração) Universidade Anhanguera, Ribeirão Preto, 2015.

QUINQUIOLO, J. M. Avaliação da Eficácia de um Sistema de Gerenciamento para Melhorias Implantado na Área de Carroceria de uma Linha de Produção Automotiva. Taubaté/SP: Universidade de Taubaté, 2002.

SOUZA, R. Metodologia para desenvolvimento e implantação de sistemas de gestão da qualidade em empresas construtoras de pequeno e médio porte. 1997, 387 p. Tese (Doutorado) - Escola Politécnica, Universidade de São Paulo. São Paulo, 1997.

WERKEMA, M. C. C. As Ferramentas da Qualidade no Gerenciamento de Processos. Belo Horizonte: Fundação Christiano Ottoni, 1995. 